\title{
The perception of motion during colinear eye movements
}

\author{
HANS WALLACH \\ Swarthmore College, Swarthmore, Pennsylvania \\ ROBERT BECKLEN \\ Sarah Lawrence College, Bronxville, New York \\ and \\ DONNA NITZBERG \\ Swarthmore College, Swarthmore, Pennsylvania
}

\begin{abstract}
When the eyes are engaged in pursuit movements, the image of a stationary object shifts on the retina, but such a target is either perceived as stationary or seems to move only little. This is the result of a compensation process called position constancy, which takes the eye movements into account. Becklen, Wallach, and Nitzberg (1984) reported that position constancy does not operate when the target undergoes a motion of its own, in a direction that differs from the direction of the eye movements. Other findings have indicated that position constancy has an effect when the target motion is colinear with the eye movements, but the accuracy with which it then operates has not been known. We measured how correctly motions that were colinear with eye movements were perceived and found that the extents of target motions were accurately perceived when they were in the same direction as the eye movement, but that position constancy showed a small, but distinct, lag when eye-movement and target motions were in opposite directions.
\end{abstract}

When the eyes move in pursuit of a moving object, the retinal images that correspond to the stationary environment shift across the retinas, but the environment appears immobile. Under the same conditions, a single stationary dot on a homogeneous background may be seen to move slightly in the direction opposite to the eye movement, but the perceived motion is much smaller than the displacement of the dot's image would warrant (Mack \& Herman, 1978). A compensation process, called position constancy, appears to operate here, preventing the displacement of the dot's retinal image from resulting in perceived motion of the dot. (Mack and Herman, 1978, presented evidence that the apparent motion of a single stationary dot during ocular pursuit does not result from an incomplete compensation but from the object-relative displacement between the dot and the object whose motion guides the eyes' pursuit movement.)

What happens to a target that is not stationary but moves while the eyes are engaged in ocular pursuit? In that case, the displacement of the target's image on the retina is the resultant of two displacements, one caused by the target's motion and the other caused by the eye movement. If po-

This work was supported by Grant BNS 83-18772 from the National Science Foundation to Swarthmore College, Hans Wallach, principal investigator.

H. Wallach's mailing address is: Department of Psychology, Swarthmore College, Swarthmore, PA 19081. sition constancy operates here and compensation for the image displacement due to the eye movement takes place, the perceived motion of the target is in agreement with its objective motion. If, however, no such compensation takes place, the perceived target motion depends on the path of the target's image and is different from the objective target motion. The issue was put to a test by Becklen, Wallach, and Nitzberg (1984), who found that here the perceived target motion depended directly on the displacement of the retinal image of the target; no compensation for the image-path component that resulted from the eye movement took place. However, in all experiments by Becklen et al. (1984), the objective motion of the target formed an angle with the direction of the eye movement. No experiments were done where the target motion was parallel to the eye movement. Since it seemed possible that failure of position constancy to operate was not due to target motion, as such, but rather to the difference in the directions of the target motion and of the eye movements, it became necessary to ask whether position constancy operated when target motion and eye movements were parallel. That is what happens, for instance, when the target and the point whose motion guides the pursuit movement of the eyes, the tracked point, move on parallel paths but in opposite directions. In that case, the image displacement due to the eye movement is added to the displacement of the image of the target that is due to its objective motion. If position constancy operates here 
and the target motion is correctly perceived, the compensation for the image displacement due to eye movement takes the form of a subtraction of the image displacement due to eye movement from the total displacement of the target image. When, on the other hand, the target motion and the motion of the tracked point differ in direction, the actual image displacement is the kinematic resultant of the displacement due to target motion and the displacement due to the eye movement. In that case, compensation requires a vector analysis of the actual image displacement, where the actual image displacement and information about the direction of the eye movement are given and the direction of the target motion is the result. Thus, if compensation were to take effect here, it would have to operate quite differently from the case in which target motion and eye movement are parallel and the extent of the target image displacement, and not its direction, is at issue. The latter is also the case when the target motion and the eye movement are in the same direction, that is, when the tracked point and the target move at different rates but in the same direction. In that case, the displacement of the target image on the retina corresponds to the difference between the target motion and the motion of the tracked point. Here, too, compensation has to deal with the extent of the target image displacement and the extent of the eye movement, and vector analysis is not needed. Thus, it does not matter whether the target and the tracked point move in the same or in the opposite direction; as long as they are colinear, the compensation process deals with the extent of the target image displacement and the extent of the eye movement and does not involve a vector analysis. This difference makes it possible that position constancy operates under these colinear conditions, although compensation fails in the case of other target motions.

An incidental result of a series of experiments by Mack and Herman (1973) shows that position constancy operates under colinear conditions. Those authors investigated whether a stationary background pattern was seen to move when the eyes tracked a moving target spot in front of it or appeared to be stationary, that is, showed position constancy. Because the pattern often appeared to move in the direction opposite to the target motion, they also presented the pattern in motion, either in the direction of the target motion or opposite to it. The velocity of the pattern motion varied up to $1 \% \mathrm{sec}$, and the number of reports of the correct motion direction at each velocity was recorded. The subjects simply reported whether or not they saw the pattern move and, if so, in which of two directions. These reports made it possible to locate a range of motions in either direction where the pattern appeared to be stationary, but the results also showed that the direction of a target motion could be perceived during colinear eye movements.

Our own experiments were quite different from those of Mack and Herman (1973). We asked whether the extent of a target motion was correctly perceived when the eyes were engaged in colinear movements. The extent of the perceived target motion was measured while the eyes tracked a dot that moved either in the same direction as the target or in the opposite direction. If position constancy operates, that extent would, of course, be in agreement with the extent of the target motion. But, if it does not, and the perceived target motion depends directly on the displacement of the target image, perceived motions would be of different extents for the two colinear conditions. When the motions of the target and of the tracked dot are in opposite directions, the displacement of the target image on the retina corresponds to the sum of these motions, and so would the perceived target motion. When the motions of the target and of the tracked dot are in the same direction, the target image path corresponds to the differences between the two motions, and so would the perceived motion of the target. To demonstrate position constancy, the length of the target motion that is perceived while the moving point is tracked needs to be compared with its length perceived while the target itself is tracked.

We measured the perceived length of the target's path with an arrangement that had been repeatedly used in the past. In this arrangement, a dot moves in reciprocating motion up and down on a pattern of vertical lines that move left and right, reversing its motion direction simultaneously with that of the dot. The motion of the line pattern induces horizontal motion of the dot, which combines with the dot's real vertical motion. An oblique motion path results, whose tilt angle depends on the strength of the induced motion and the extent of the vertical dot motion. The tangent of the angle between the apparent motion path and the vertical, which is measured as a tilt estimate, has been used to measure the induced motion represented by the horizontal component of the oblique path (Wallach, Bacon, \& Schulman, 1978; Wallach, O'Leary, \& McMahon, 1982). In our experiments, the arrangement was used to measure the apparent extent of the vertical target motion represented by the vertical component of the oblique path, that is, as the cotangent of the estimated tilt angle. ${ }^{1}$ In our first experiment, the tracked point and the target moved in opposite directions.

\section{EXPERIMENT 1}

\section{Method}

Subjects. Twenty-one paid undergraduates served as subjects.

Equipment. The apparatus employed by Becklen et al. (1984) in their Experiment 3 was slightly modified. A motor-driven mechanism turned two different mirrors through small angles in simultaneous reciprocating motions. One of them turned about a vertical axis and reflected a projector beam that carried the line pattern. The beam came to a focus on a large translucent screen, where it moved left and right when the mirror turned alternately clockwise and counterclockwise. A second mirror turned about a horizontal axis. It shifted the beam of a second projector, which carried a small light dot back and forth in the vertical dimension and, at the same time, turned it upwards. This beam encountered a beam splitter, which reflected a portion of the beam toward the screen, where the dot moved up and down when the second mirror turned. The other portion of the beam continued upward, passed through a Dove prism that reversed its motion direction, and, fi- 
nally, was reflected toward the screen by still another mirror. This arrangement provided two dots that moved vertically but always in opposite directions. One of the dots moved with a larger excursion than the other because its beam traversed a larger distance before it reached the screen. The lantern that projected the dot, which in due course became two, was so focused that the dots on the screen lacked equal sharpness.

Display. One of the dots moved up and down through a distance of $11 \mathrm{~cm}$; the excursion of the other was $8.5 \mathrm{~cm}$. Their motion paths were $5 \mathrm{~cm}$ apart, and the lower end of the shorter path was $4 \mathrm{~cm}$ lower than the other. The dots measured about $.7 \mathrm{~cm}$ across. The line pattern was $96 \mathrm{~cm}$ high and $65 \mathrm{~cm}$ wide, and the lines, brighter on the darker ground, were $1 \mathrm{~mm}$ thick and $2 \mathrm{~cm}$ apart. The horizontal excursion of the lines was $9 \mathrm{~cm}$. All motions were reciprocating and simple harmonic, and all reversed direction simultaneously. One motion cycle took $8.6 \mathrm{sec}$.

The subject gave estimates of the tilt angle of the apparent motion path of the target dot using a device that had been employed by Wallach et al. (1978) and Wallach et al. (1982). It consisted of a white rod, $57 \mathrm{~cm}$ long, that was perpendicularly attached to a horizontal shaft so that it could be given any orientation in a vertical plane. The rod was visible against a square black surface and was located to the subject's left, with its rotation plane forming a right angle with the screen, at a distance of $75 \mathrm{~cm}$ from the subject's eyes.

Procedure. The subject, seated on an adjustable chair so that the center of the display was at eye level, was asked to follow one of the dots with his or her eyes and then draw, on a large paper pad, the direction of the motion path of the other dot. The subject made this observation and did a drawing three times. This procedure gave the subject practice with the difficult task of tracking one dot and observing the slope of the other dot's path. Then the subject was instructed in the use of the tilt-estimation device, and the measurement began. After the subject had once more observed the target motion, a small table lamp illuminating the white rod was turned on. The subject turned to face the rod and set it to an orientation that duplicated the tilt angle of the observed motion path. The angle that the rod formed with the vertical was recorded. This procedure was followed twice, once with the initial orientation of the rod horizontal and once with it vertical. The average of the two settings became the subject's tilt-estimation score. After the two tilt estimates were obtained for one dot, the same procedure was followed for the other dot: Three practice observations were made, and the perceived tilted path was drawn each time. Then the subject gave the two tilt estimates. Then, without further practice observations, a second set of tilt estimates was obtained for each target dot, in the same order as before.

After tilt-estimation scores had thus been obtained, twice for each dot under the experimental condition in which one dot was the target for which tilt estimates were given while the other dot was tracked, tilt estimates were obtained for the paths of the dots when they were themselves tracked. The tilt-estimation scores that resulted served as controls, for they represented the perceived extent of the target motion when that motion was given directly, by ocular pursuit, rather than by the compensation process or by the actual image path.

This procedure was always followed; only the order in which the two dots served as target and the initial position of the test rod was varied.

\section{Results}

The mean cotangents of the estimated tilt angles of the short and long motion paths are listed in Table 1 . The results of the control condition in which tilt estimates for the paths of the tracked dots were given are listed in columns $c$ and $f$ of the table. Under the assumption that the motion of the line pattern, which amounted to $9 \mathrm{~cm}$, was fully effective in causing horizontal induced motion of the dots, the mean cotangent values listed corresponded to vertical components of the estimated tilts, amounting to 8.4 and $11.8 \mathrm{~cm}$. The agreement of these values with the lengths of the actual dot paths of 8.5 and $11.0 \mathrm{~cm}$ shows that the line-pattern motion was indeed almost fully effective. To ascertain whether the mean cotangents obtained in the experimental conditions and listed in columns $\mathrm{a}, \mathrm{b}, \mathrm{d}$, and e denote constancy or whether they correspond to the target dot's image paths, they were compared with the results of the control condition, on the one hand, and with the cotangent of the tilt angle that would result if its perceived vertical component depended on the target dot's image path, on the other. Since, in this experiment, the tracked dot and the target dot moved in opposite directions, the target's image path corresponded to the sum of the two motion paths, which was $19.5 \mathrm{~cm}$. Because it can be assumed that the horizontal motion of the line pattern was almost fully effective in causing horizontal induced motion of the target dot, the horizontal component of the target's motion path corresponded to the 9$\mathrm{cm}$ extent of the lines' motion. Assuming, therefore, that a horizontal component of $9 \mathrm{~cm}$ goes with the $19.5-\mathrm{cm}$ long motion that corresponds to the vertical image path, the cotangent for the tilt angle to be expected if there were no constancy was $19.5 / 9=2.167$.

The mean cotangent values obtained in the experimental conditions (E) were much closer to the means obtained

Table 1

Mean Cotangents of Estimated Tilt Angles Representing the Lengths of the Vertical Path of a Target Dot, When the Other Dot is Tracked (Experimental Condition E) or When the Target Dot is Tracked (Control Condition C)

\begin{tabular}{|c|c|c|c|c|c|c|}
\hline & \multicolumn{3}{|c|}{ Estimates for Short Path } & \multicolumn{3}{|c|}{ Estimates for Long Path } \\
\hline & \multicolumn{3}{|c|}{ Path Tracked } & \multicolumn{3}{|c|}{ Path Tracked } \\
\hline & $\begin{array}{c}\text { Long (E) } \\
\mathbf{a}\end{array}$ & $\begin{array}{c}\text { Long (E) } \\
\mathrm{b}\end{array}$ & $\begin{array}{c}\text { Short (C) } \\
\mathrm{c}\end{array}$ & $\begin{array}{c}\text { Short (E) } \\
\mathrm{d}\end{array}$ & $\begin{array}{c}\text { Short (E) } \\
\mathrm{e} \\
\end{array}$ & $\begin{array}{c}\text { Long (C) } \\
\mathrm{f}\end{array}$ \\
\hline $\begin{array}{l}\text { Mean } \\
\text { SD }\end{array}$ & $\begin{array}{r}1.230 \\
.589\end{array}$ & $\begin{array}{r}1.178 \\
.606\end{array}$ & $\begin{array}{l}.938 \\
.362\end{array}$ & $\begin{array}{r}1.563 \\
.483\end{array}$ & $\begin{array}{r}1.496 \\
.302\end{array}$ & $\begin{array}{r}1.312 \\
.464\end{array}$ \\
\hline \multicolumn{7}{|c|}{ Different from: } \\
\hline $\begin{array}{l}C \\
2.167 \\
\end{array}$ & $\begin{array}{r}+.292 \\
-.937 \\
\end{array}$ & $\begin{array}{r}+.240 \\
+.989 \\
\end{array}$ & & $\begin{array}{l}+.251 \\
-.604 \\
\end{array}$ & $\begin{array}{r}+.184 \\
-.671 \\
\end{array}$ & \\
\hline
\end{tabular}


in the control condition (c) than to the value of 2.167. The differences between the latter value and the means for the experimental conditions were highly significant, with $t(20)=7.29$ and 7.48 in case of the short path and $t(20)=5.73$ and 10.18 in case of the long path. Although all mean cotangents that were obtained in the experimental condition were larger than the means of the control conditions, these differences were not as large as those between the means of the experimental conditions and the value 2.167 . For easy comparison, the differences between the means for the experimental condition and for the control condition are listed in the third row of Table 1 and the differences between the means for the experimental condition, and the value of 2.167 expected if there were no constancy are listed in the fourth row. They show the latter differences to be much larger, so much so that the conclusion is warranted that position constancy did operate. But it operated with a distinct lag, with the differences between the mean cotangents of the experimental conditions and those of the control conditions also being significant, with $\mathrm{t}(20)=3.14$ and 2.47 for the means for the short path and $t(20)=2.91$ and 2.35 for those of the long path.

\section{EXPERIMENT 2}

\begin{abstract}
Method
Subjects. Fourteen paid undergraduates served as subjects.

Display. Our second experiment closely resembled the first, except that the dots always moved in the same direction. The motion direction of one of the dots was reversed by turning the Dove prism through which the beam passed. Since the dots now moved in the same direction, the image path of the target dot was shorter than the extent of the motion of either dot, since it corresponded to the difference in length of the two motion paths. Because it was short, the tilt angle of the resultant oblique motion to be expected if constancy did not operate would be quite large. To avoid having perhaps to deal with extreme tilts, we shortened the excursion of the line pattern to $6 \mathrm{~cm}$ and thereby diminished the horizontal component of the perceived motion path. The extents of the dot's motion paths were 10.8 and $8.1 \mathrm{~cm}$ and, with the difference between the extents of the paths amounting to $2.7 \mathrm{~cm}$, the expected tilt angle, if constancy did not operate, would be $66^{\circ}$ and the expected cotangent .450 . The distance between the paths was $4.8 \mathrm{~cm}$, and the lower end of the longer path was $1 \mathrm{~cm}$ higher than the lower end of the shorter path.
\end{abstract}

Procedure. The procedure was the same as in Experiment 1.

\section{Results}

The mean cotangents of the estimated tilt angles are listed in Table 2 in the six columns under the headings
"Experiment 2." In the case of the short motion path, there was no difference between either one of the means obtained in the experimental condition in which the subject's eyes tracked the long path and the mean from the control condition in which the target dot itself was tracked. In the case of the long path, the differences between experimental and the control mean were in the direction opposite to what would be expected from a lag in constancy. They were not statistically significant, since they fell well within the .05 confidence limits of \pm .326 for the mean difference scores.

Before we can conclude that, at least for the shorter target motion, constancy was complete when the target moved in the same direction as the eye movement, we had to show that, in the control condition, the presence of the other moving dot had no effect on the perceived target motion. This was done in our last experiment.

\section{EXPERIMENT 3}

\section{Method}

Subjects. Twelve undergraduates served as subjects.

Procedure. Only the control conditions of the previous experiments were used. A further control condition was added in which the other dot was eliminated when the tilt estimates for the tracked dot were obtained. The subject started by making two drawings of the sloping path of a tracked dot, observed in the presence of the other moving dot. Then two tilt estimates were obtained for the identical condition. Following this, tilt estimates were obtained for the other dot, in the presence of the first dot. Finally, tilt estimates were obtained for each dot alone. This sequence was always followed; only the order in which the two dots served as target and the initial position of the test rod were varied.

\section{Results}

The mean cotangents of the estimated tilt angles of the tracked target dots obtained in the repeated control condition (C) and the new control condition (CC) are listed under the headings "Experiment 3"' in Table 2. They are virtually the same, and, since the means for the repeated control conditions obtained in Experiment 3 were in excellent agreement with those obtained in Experiment 2 under the same conditions, one can conclude that the presence of the other dot motion in the control condition of Experiment 2 had no effect on the tilt estimates that represented the perceived path of the tracked target dot. Therefore, the conclusion that constancy was complete under the conditions of Experiment 2, in which the target and the tracked point moved in the same direction, is justified.

Table 2

Mean Cotangents of Estimated Tilt Angles Representing the Lengths of the Vertical Path of a Target Dot When the Other Dot Is Tracked (E), When the Target Dot Is Tracked and the Other Dot is Present (C), or When the Target Dot Is Tracked and the Other Dot is Absent (CC)

\begin{tabular}{|c|c|c|c|c|c|c|c|c|c|c|}
\hline & \multicolumn{5}{|c|}{ Estimates for Short Path } & \multicolumn{5}{|c|}{ Estimates for Long Path } \\
\hline & \multicolumn{3}{|c|}{ Experiment 2} & \multicolumn{2}{|c|}{ Experiment 3} & \multicolumn{3}{|c|}{ Experiment 2} & \multicolumn{2}{|c|}{ Experiment 3} \\
\hline & $\mathrm{E}$ & $\mathbf{E}$ & $\mathrm{C}$ & $\mathrm{C}$ & $\mathrm{CC}$ & $E$ & $\mathrm{E}$ & $\mathrm{C}$ & $\mathrm{C}$ & $\mathrm{CC}$ \\
\hline $\begin{array}{l}\text { Mean } \\
\text { SD }\end{array}$ & $\begin{array}{l}.956 \\
.569\end{array}$ & $\begin{array}{r}.845 \\
493\end{array}$ & $\begin{array}{r}.928 \\
255\end{array}$ & .919 & $\begin{array}{r}.927 \\
162\end{array}$ & $\begin{array}{r}1.598 \\
630\end{array}$ & $\begin{array}{r}1.551 \\
553\end{array}$ & $\begin{array}{r}1.392 \\
414\end{array}$ & $\begin{array}{r}1.364 \\
395\end{array}$ & $\begin{array}{r}1.354 \\
267\end{array}$ \\
\hline
\end{tabular}




\section{DISCUSSION}

We found that position constancy was complete when, in Experiment 2, the motion directions of the tracked and target dots were the same, whereas there was a small, but significant, lag in constancy when, in Experiment 1, the target motion and the eye movement were in opposite directions. This lag cannot be attributed to an underregistration of the tracking eye movement (cf. Mack \& Herman, 1973), because such an underregistration would also have affected the control condition in which the target itself was tracked, since the control condition served to define this lag. The lag seems, rather, to be inherent in the compensation process. Since it is not known how that process is acquired, we cannot offer an explanation for this aspect of our results.

In these experiments, we measured the extents of the perceived target motions indirectly, by combining them with an induced motion, and had the tilts of the resultant motion paths represent those extents. This was necessary because it is extremely difficult to give actual estimates of motion extents while the eyes track the motion of a second dot. The relative displacement between the target and the tracked dot has an effect on the estimates, which should be based solely on subject-relative cues. Our technique for comparing the perceived extents of the target motion under the experimental and control conditions presupposes that the effect of the motion of the line pattern on the horizontal component of the tilted motion path is the same under the two conditions. It is assumed that it does not matter whether the tracking movements of the eyes are longer or shorter, or how the target motion is given. We do believe that the vertical motion of the dots has no influence on the induced motion they also undergo. The induced motion results from the configurational change between the dots and their background, whereas their vertical motions are given by image displacement or ocular pursuit. ${ }^{2}$ These stimulus conditions evoke perceptual processes that are very different from each other. Induced motion is a matter of form perception and thus takes a different course from that of the evaluation of eye movements or of image displacement. The processes develop independently of each other-including the operation of position constancy - until they eventually combine to form the resultant motion path (Wallach, Becklen, \& Nitzberg, 1985).

There is also experimental evidence that the real dot motion does not alter the induced-motion component. In Experiment 3, the mean cotangents of the estimated angles of the target motions were the same whether a second moving dot was present or not. Compare each mean under $\mathrm{C}$ with the corresponding mean under $\mathrm{CC}$ in Table 2.

Our results raised an interesting question: Why does position constancy operate when the motion that a target undergoes when the eyes are engaged in a pursuit movement is colinear with the motion of the tracked point, but does not operate when target motion and eye movement have different directions? In the present case, in which target motion and the motion of the tracked point were colinear, compensation consisted of a subtraction of the image displacement due to the eye movement from the total displacement of the target image, or in a corresponding addition. When the direction of the target motion and the direction of the motion of the tracked point differ, the actual image displacement is the kinematic resultant of the retinal displacements that represent these two motions, and compensation for the image path component caused by the eye movement would have amounted to performing a vector analysis. The failure of compensation to operate in the latter case may well mean that such a vector analysis cannot be performed. For many years, the operation of a vector analysis seemed to be required by the motions perceived in certain displays discovered by Johansson (1950), but recently Wallach et al. (1985) have proposed an explanation of Johansson's observations that does not imply a vector analysis.

\section{REFERENCES}

Becklen, R., W Allach, H., \& Nitzberg, D. (1984). A limitation of position constancy. Joumal of Experimental Psychology: Human Perception and Performance, 10, 713-723.

Johansson, G. (1950). Configurations in event perception. Uppsala, Sweden: Almqvist \& Wiksells.

Mack, A., \& Herman, E. (1973). Position constancy during pursuit eye movements: An investigation of the Filehne illusion. Quarterly Journal of Experimental Psychology, 25, 71-84.

Mack, A., \& Herman E. (1978). The loss of position constancy during pursuit eye movements. Vision Research, 18, 55-62.

Wallach, H., Bacon, J., \& Schulman, P. (1978). Adaptation in motion perception: Alteration of induced motion. Perception \& Psychophysics, 24, 509-514.

WALLACH, H., BeCkLEN, R., \& NitzBeRG, D. (1985). Vector analysis and process combination in motion perception. Joumal of Experimental Psychology: Human Perception and Performance, 11, 93-102.

Wallach, H., O'Leary, A., MCMaHon, M. L. (1982). Throe stimuli for visual motion perception compared. Perception \& Psychophysics, 32, 1-6.

\section{NOTES}

1. Since the horizontal induced motion of the dot and its real vertical motion formed a right angle, the resultant perceived motion of the dot formed the hypotenuse of a rectangular triangle, with the two components forming the two sides. Because the estimated tilt angle is the angle between the hypotenuse and the vertical side, the two components form its tangent or cotangent. With the motion of the line pattern the same in all our experiments, the extent of the induced motion was assumed to be also the same throughout. In that case, the tilt angle varies with the extent of the vertical motion, which is therefore proportional to the cotangent of the tilt angle.

2. It is important here that the background pattern consisted of vertical lines and provided no reference points for the dots' motions. Therefore, there was no sensory basis for an interaction between the displacement on the dots and the line motion.

(Manuscript received January 15, 1985; revision accepted for publication July 8, 1985.) 